\title{
La escuela como instauradora de la Ley en la trama edípica
}

AS IT RESTORES SCHOOL OF LAW IN THE OEDIPAL FRAME

A ESCOLA COMO INSTAURADORA DA LEI NA TRAMA EDÍPICA

Daniela Belén López* / danyb186@hotmail.com

Flavia Andrea Navés** / flaviaandreanaves@gmail.com

\section{Resumen}

El presente trabajo hace parte del "Proyecto Ubacyt 2011-2014: Actitudes de estudiantes universitarios de grado y de posgrado de la Facultad de Psicología de la Universidad de Buenos Aires respecto de la ética profesional", dirigido por la Dra. Elizabeth B. Ormart. Como trabajadores de la educación sabemos que cuando un chico ingresa a la escuela se establece un interjuego de tres partes: la familia, el alumno y el docente; es en este espacio donde se ponen en juego los deseos, expectativas, temores y conflictos de cada uno. El objetivo del presente escrito es realizar un análisis sobre la función de la escuela como instauradora de la Ley en la constelación familiar.

\section{Summary}

Thpart of the "Project Ubacyt: Attitudes of universitye present work makes 2011-2014 a students of degree and of posgrado of the Faculty of Psychology of the University of Buenos Aires I concern of the professional ethics ", directed by the Dra. Elizabeth B. Ormart. Since workers of the education we know that when a boy enters to the school there is established an intergame of three parts: the family, the pupil and the teacher; it is in this space where there are brought into play the desires, expectations, dreads and conflicts of each one. The aim of the written present is to realize an analysis on the function of the school as instauradora of the Law in the familiar constellation.

\section{Resumo}

O presente trabalho faz parte do "Projecto Ubacyt 2011-2014: Atitudes de estudantes universitários de grau e de posgrado da Faculdade de Psicologia da Universidade de Buenos Aires respecto da ética profissional", dirigido pela Dra. Elizabeth B. Ormart. Como trabalhadores da educação sabemos que quando um garoto ingressa à escola se estabelece um interjuego de três partes: a família, o aluno e o docente; é neste espaço onde se põem em jogo os desejos, expectativas, temores e conflitos da cada um. O objectivo do presente escrito é realizar uma análise sobre a função da escola como instauradora da Lei na constelação familiar.
Palabras clave

Interjuego, familia, alumno, docente

Key words

Intergame, family, pupil, teacher

Palavras chave

Interjuego, família, aluno, docente

* Becaria de la Dirección de Políticas Socioeducativas. Subsecretaría de Educación e inclusión Social. Municipalidad de Avellaneda. Docente para adultos de Psicología (Plan FINES), en 3 Centros del Distrito Avellaneda: Centro UNDAV, Centro Juventud, y Dirección Social Martín Güemes. Estudiante avanzada del Prof. Enseñanza Media y Superior en Psicología. Universidad de Buenos Aires.

** Profesora de Matemática y Astronomía en escuelas media. Partido de La Matanza. Provincia de Buenos Aires. Ayudante Ad-Honorem Cátedra I. Psicología, Ética y Derechos Humanos. Facultad de Psicología. Universidad de Buenos Aires. Estudiante avanzada de la Lic. en Psicología. Facultad de Psicología Universidad de Buenos Aires.

Fecha de recepción: 18 de mayo de 2012 / Fecha de aprobación: 8 de junio de 2012 


\section{Introducción}

Las cuestiones que animan este trabajo son de índole muy diversa, en principio el hecho de que la realidad nos dice que la escuela y la familia son instituciones que han sufrido grandes modificaciones a través del tiempo que, sin embargo, se interrelacionan. Es por ello que para comenzar presentaremos el caso que dio origen al presente escrito $y$, a partir del mismo, trataremos de reflexionar sobre la posición que ambas instituciones mantienen frente a la necesidad de consolidar el vínculo existente entre ellas, sin dejar de lado su estrecha relación con la construcción de subjetividades. Por último, intentaremos pensar, con una mirada ética, acerca de las consecuencias en la subjetividad de quienes transitan por la trayectoria educativa que incita esta relación.

\section{¿Educando a los niños o educando al alumno?}

Las prácticas escolares no son ajenas al profundo problema socio-cultural que atañe a la época contemporánea. El malestar presente en las mismas nos indica la existencia de una crisis general que tiene sus raíces en las transformaciones sociales que se desarrollan de manera vertiginosa en la actualidad. En este contexto, la trayectoria escolar ha sufrido una transformación de sentido, por lo que se hace necesario reflexionar acerca de las posiciones subjetivas que el experimento escolar moderno concebía y que ya no tienen lugar.

En la actualidad, ser alumno ya no es sinónimo de ser niño, un adulto también puede ocupar ese lugar. Del mismo modo, la escuela y los docentes han pasado a cumplir funciones para las que no fueron preparados: los docentes trabajan de psicólogos, trabajadores sociales, atienden el comedor, etc., esto es válido para los alumnos ${ }^{1}$ que transitan la trayectoria escolar. Sin embargo, a partir de la crisis del 2001, son cada vez más los niños que se encuentran fuera de este recorrido, obligatorio sólo en teoría, pues su práctica está lejos de cumplir con los mínimos estándares.

A partir de estos cambios, el Estado ha intentado dar respuesta a las nuevas necesidades sociales, promoviendo estrategias que permitan la inserción escolar de la franja

1 La notación @ se utiliza en muy variados ámbitos para referirse a alumnos y alumnas o niños y niñas, es decir a los dos sexos. etaria que supera los límites de la edad permitida, para terminar sus estudios en las escuelas medias. El Plan FINES se ha desarrollado con este objetivo, y es un programa de alcance nacional que funciona en todas las jurisdicciones y contempla la posibilidad de que quienes no hayan podido concluir sus estudios puedan hacerlo, independientemente de la edad que tengan.

Es dentro de este marco donde tiene lugar el caso que nos convoca y al que le hemos dado el nombre de "Caso M", en donde madre e hijo son incorporados juntos al plan, ya que las condiciones de cursada (haber finalizado el primario y ser mayor de 18 años) se lo permiten a ambos.

\section{Viñeta: caso $M$}

M y su madre J se presentan juntos el primer día de la clase de Psicología en su condición de alumnos. Al momento de la presentación de cada uno de los integrantes de la clase, J se presenta como la mamá de $\mathrm{M}$, obviando decir su nombre. A partir del desarrollo de las clases, en la materia Psicología, ella refuta o confirma todos los temas propuestos por la docente (etapas evolutivas, diferencias de género, problemáticas sociales, etc.), justificando sus respuestas a partir de lo que considera que su hijo ha experimentado (o lo ha vivido) de ese modo o no.

Cuando se propuso, en clase, realizar el primer trabajo práctico, M faltó y J entregó una fotocopia del que había realizado ella, firmándola con el nombre de su hijo. Durante la cursada, cuando se Ilama al alumno para realizar las devoluciones de las distintas entregas, J se para al costado de su hijo para escuchar la nota y las correcciones realizadas por el docente.

Así mismo, si el docente le pide silencio a $\mathrm{M}$, por ejemplo, cuando conversa mucho en clase, J se pone muy seria, demostrando inconformidad a través de sus expresiones verbales y no verbales. Cabe aclarar que J es un referente importante en el barrio donde se dictan dichas clases, y además es muy querida por el resto de los alumnos, para quienes es natural el comportamiento que tiene con su hijo. Por su lado, M. tiene dificultades en el aprendizaje, no tolera equivocarse y mira a su madre cuando se le pregunta algo que no sabe; ella contesta en su lugar. 


\section{Dime de dónde vienes...}

Cada vez en mayor medida los docentes de la escuela media solemos enunciar quejas sobre la falta de motivación e interés de los padres respecto de la educación de sus hijos. Sin embargo, también existen casos, como el que motivó el presente trabajo, donde estos se presentan ante las autoridades educativas como omnipresentes y muy resistentes a que su hijos reciban una educación diferente a la que fue transmitida por su núcleo familiar primario.

Cuando un niño ingresa a la escuela se establece un interjuego de tres partes: familia, alumno y docente. Es en este espacio donde se da la interacción de los deseos, expectativas, temores y conflictos de cada uno. Tanto la familia como la escuela son instituciones que han sufrido numerosos cambios en las últimas décadas, y en este contexto no es extraño escuchar a los docentes justificar la mala conducta o las dificultades en el aprendizaje de un alumno sobre la base de su familia; generando una especie de profecía autocumplida en donde el niño o el adolescente reproducirá las pautas de comportamiento de la familia o el contexto cultural del que procede; sin embargo, vale la pena recordar, junto a Isabelino Siede (2006), que:

La escuela se ha dedicado a enseñar cómo debe ser una familia según los cánones de normalidad establecidos por el higienismo y las corrientes que provenían de las ciencias sociales de las primeras décadas del siglo XX.

Frente a esta herencia, surge la necesidad de reflexionar acerca de cómo trabajar con nuestras representaciones sociales acerca del "debería ser" de las familias de nuestros alumnos, de modo que este imaginario no dificulte nuestro trabajo con ellos; en relación con esto, Arrué nos dice que:

Es necesario tender puentes entre familias devastadas y escuelas desbordadas, entre los adultos y los niños. Reflexionar sobre las propias prácticas permitirá correrse de esa mirada culpabilizadora de las familias, tan cristalizada en las escuelas (Arrué, 2009).

Es una decisión política e ideológica trabajar a partir de la diversidad de alumnos y de grupos familiares, considerando que una de las funciones principales de la educación es dar herramientas para comprender la realidad social, comprendiendo que existen otros modos de hacer, pensar, sentir y creer, más allá del propio, con encuentros centrados en las potencias (y no en las dificultades), estableciendo nuevos modos de relación entre alumnos, familias y escuelas, generando una inclusión real de todos e invirtiendo el sentido normalizador y elitista con el que la escuela ha sido creada.

\section{La función materna, entre la renuncia y la voracidad}

Asistimos en la actualidad a una transformación que tiene lugar en la estructura familiar tradicional; por citar algunos ejemplos, el tiempo que sus miembros permanecen unidos ha disminuido significativamente, produciéndose cambios en el estilo comunicacional entre sus integrantes, al tiempo que los lugares ocupados tradicionalmente por cada uno de ellos, también se encuentran trastocados.

Sin embargo, la institución familiar tiene por función la transmisión del capital simbólico que le garantiza al cachorro humano su iniciación en el lenguaje, asignándole un lugar en la cadena generacional y ubicándolo al mismo tiempo en un sistema de intercambio cultural. Para ser exactos, diremos que una familia, sin importar su constitución, sostiene relaciones singulares que garantizan la transmisión y el sostén de un núcleo social no anónimo y singular.

En el caso que nos convoca puede apreciarse la relación singular que mantienen madre e hijo. Si dejamos que estas nominaciones queden impregnadas por las diversas formas de opinión quedaríamos atrapados en un callejón sin salida. Es por ello que intentaremos ir más allá de cualquier sesgo ideológico, cuando nos preguntamos ¿Qué es una madre? ¿Puede una madre renunciar a su lugar? ¿Qué es un padre? ¿Puede un hijo poner un límite a la voracidad materna? ¿Cuál es la función de la institución? Sobre este punto nos detendremos un momento e intentaremos reflexionar sobre estos interrogantes apoyados en la teoría psicoanalítica.

La función de la institución educativa, dentro de la relación madre-hijo, parecería corresponderse con la función del padre real en el segundo tiempo del complejo de Edipo. El padre real, como lo define J. Lacan, realiza la operación de corte, haciendo valer su deseo e instaurando su Ley, obligando a una doble renuncia, la de la madre, aquella que puede ceder y 
puede renunciar a ese niño para que no quede capturado en el lugar de falo materno, y la del hijo. Es decir que la institución educativa, y quienes la representan, instituye la función paterna entre M y J; parafraseando a Lacan, podríamos decir que le pone el palo a la boca del cocodrilo para limitar el deseo voraz de la madre (J).

\section{Consideraciones finales}

Las transformaciones sociales que caracterizan a la sociedad contemporánea se ven reflejadas en la escuela y la familia; a pesar de que ambas conservan la peculiaridad de ser quienes transmiten la cultura,

\section{Referencias}

Arrue. (2009). Un piano en el puente. Experiencias educativas favorecedoras de inclusión. En Elichiry, N. (Comp). Inclusión educativa. Investigaciones y experiencias en Psicología Educacional. Buenos. Aires: JVE.

Baquero, R. (2001). La educabilidad bajo sospecha. Cuaderno de Pedagogía. No 9. Rosario.

Baquero, R. (2007). Los saberes sobre la escuela. Acerca de los límites de la producción de saberes sobre lo escolar. Ficha CEP.

Baquero, R., Tenti, Fanfani, E., y Terigi, F. (2004). Educabilidad en tiempos de crisis. Condiciones sociales y pedagógicas para el aprendizaje escolar. Nuevos paradigmas. Educabilidad en tiempos de crisis, 16 (168). Buenos Aires: Ed. Novedades Educativas.

Lacan, J. (1970). Seminario 5. Las formaciones del inconciente. Buenos Aires: Nueva visión.

Lacan, J. (1993). Dos notas sobre el niño. Intervenciones y textos 2. Buenos Aires: Manantial. hay un desfase de las funciones que cada institución debería cumplir. El caso M demuestra cómo estas transformaciones sociales afectan la función de cada una de las instituciones; y permite ejemplificar de qué manera la escuela podría cumplir con la función paterna, allí donde algo de la misma ha fallado o está ausente, poniéndole un freno al deseo voraz de la madre y posibilitando, de este modo, un mayor grado de libertad para los alumnos que en ella habitan, pasando a cumplir de este modo muchas más funciones que la mera transmisión de conocimientos enciclopédicos, y convirtiéndose en una verdadera herramienta de transformación subjetiva y social.
Lévi-Strauss, C. (1985). Las estructuras elementales del parentesco. Barcelona: Planeta-Agostini.

Michel Fariña, J., y Gutiérrez, C. (2001). La encrucijada de la filiación. Tecnologías reproductivas y restitución de niños. Buenos Aires: Editorial Lumen.

Ormart, E. (2011). ¿Cómo escapar del superyó materno? El sigma. Obtenido desde, http://www.elsigma.com/site/detalle. asp? IdContenido $=12212$

Siede, I. (2006). Vínculos escuela y familia. 12(ntes) papel y tinta para el día a día en la escuela. Obtenido en Septiembre de 2006 , desde, http://www.google.com.ar/url?sa=t\&rct=j\&q=escuela $\% 20$ y $\% 20$ familias $\% 2012$ ntes \& source $=$ web \& cd $=1 \&$ sqi $=2$ \&ved=0CB4QFjAA\&url=http $\% 3 \mathrm{~A} \% 2 \mathrm{~F} \% 2 \mathrm{Fwww} .12$ ntes. com\%2Frevista\%2Fnumero07.pdf\&ei=1GnVTonKFYS2twfB7_Cv Ag\&usg=AFQjCNGoM72pmdHfl6b3b7IM7UPByxkOPQ 


\section{Diálogo del conocimiento}

Un primer punto a destacar es el desfasaje entre la representación social que tienen las instituciones educativas de la familia y el modo en que las familias se perciben a sí mismas. Existe una brecha entre las demandas de las instituciones a las familias y las posibilidades de éstas de adecuarse a esas demandas. Las escuelas quieren familias ideales con escasos problemas y éstas no son las más comunes. En el programa FINES que se desarrolla en Argentina desde el año 2008 se busca llegar a familias que han tenido obstáculos para lograr la permanencia de sus miembros en el sistema educativo. Familias que no han podido sostener, apoyar, apuntalar a sus integrantes, las familias con mayores dificultades y por consiguiente, las más alejadas de todo ideal. El caso particular que es comentado en el artículo de las Profesoras Navés y López es el de una madre que no pudo terminar sus estudios secundarios y su hijo, que tampoco lo logró. Ambos son incluidos en el programa FINES. Las falencias son muchas y entre ellas, se refieren puntualmente a la dificultad de separar la dinámica familiar de la escolar. La madre no puede dejar de ser madre y el hijo no puede dejar de ser hijo. Para poder aprender en las instituciones educativas es central el trabajo sostenido en la autonomía progresiva. Pasaje que va de los vínculos primarios a los secundarios, de la endogamia a la exogamia, del aprendizaje asistido al autoaprendizaje. En esta viñeta, encontramos que la intervención docente resulta insuficiente y apela a una instancia que funcione como un tercero interviniente.

En el caso particular que las autoras analizan encontramos que:

- La madre no puede abandonar el rol de madre y ubicarse en el lugar de alumna.

- El hijo no puede salirse de este lugar, ya que la madre exige en sus intervenciones que él quede ubicado allí.

- La docente de psicología encuentra que este modo de vinculación funciona como un obstáculo para el aprendizaje del hijo y de la madre.

- La institución debería intervenir separando a la madre del hijo. Ubicando al hijo en otro curso y de no ser posible, interviniendo para clarificar con los protagonistas los alcances y límites de sus roles familiares y la necesidad de operar desde otro lugar.

Por otro lado, la visión empobrecida de los docentes sobre los alumnos funciona como un obstáculo para el aprendizaje. Las profecías de fracaso autocumplidas en el terreno educativo provocan obstáculos para el éxito académico y consecuentemente, para la movilidad social.

La escuela en tanto institución social es un ámbito reglado, en el que los lugares y las funciones son la estructura que permite los aprendizajes. Si no existieran pautas, normas y leyes en la institución escolar sería imposible el aprendizaje. El aprendizaje exige libertad y creatividad que pueden darse cuando ciertas variables contextuales son estables. Constantes estructurales que funcionan como telón de fondo sobre el que se pueden desplegar encuentros educativos centrados en las potencialidades y no en las dificultades.

Elizabeth Beatriz Ormart 